\begin{tabular}{|c|c|c|}
\hline Case Reports in & \multicolumn{2}{|c|}{ Case Rep Gastroenterol 2018;12:671-678 } \\
\hline Gastroenterology & $\begin{array}{l}\text { DOI: 10.1159/000494553 } \\
\text { Published online: November 22, } 2018\end{array}$ & $\begin{array}{l}\text { (c) } 2018 \text { The Author(s) } \\
\text { Published by S. Karger AG, Basel } \\
\text { www.karger.com/crg }\end{array}$ \\
\hline & $\begin{array}{l}\text { This article is licensed under the } \mathrm{Cr} \\
\text { International License (CC BY-NC) (ht } \\
\text { Usage and distribution for commercial }\end{array}$ & $\begin{array}{l}\text { nons Attribution-NonCommercial } 4.0 \\
\text { ger.com/Services/OpenAccessLicense). } \\
\text { uires written permission. }\end{array}$ \\
\hline
\end{tabular}

\title{
An Extragastrointestinal Stromal Tumor Originating from the Pancreas
}

\author{
Seong Jae $\mathrm{Yeo}^{\mathrm{a}}$ Chang Min Cho ${ }^{\mathrm{a}}$ Hyung Jun Kwon ${ }^{\mathrm{b}}$ Seung Hyun Cho \\ Gab Chul Kim ${ }^{c}$ An Na Seo ${ }^{d}$ Han Ik Bae $^{d}$ \\ ${ }^{a}$ Department of Internal Medicine, School of Medicine, Kyungpook National University, \\ Kyungpook National University Chilgok Hospital, Daegu, South Korea; ${ }^{b}$ Department of \\ Surgery, School of Medicine, Kyungpook National University, Kyungpook National \\ University Chilgok Hospital, Daegu, South Korea; 'Department of Radiology, School of \\ Medicine, Kyungpook National University, Kyungpook National University Chilgok \\ Hospital, Daegu, South Korea; ${ }^{d}$ Department of Pathology, School of Medicine, Kyungpook \\ National University, Kyungpook National University Chilgok Hospital, Daegu, South Korea
}

\section{Keywords}

Gastrointestinal stromal tumor · Pancreas · CD117 · C-kit protein

\begin{abstract}
Gastrointestinal stromal tumor (GIST) is the most common mesenchymal neoplasm of the gastrointestinal tract (GIT). In fewer than $5 \%$ of cases, GIST originates primarily from outside the GIT. The occurrence of GIST originating from the pancreas is rare. Sometimes, neuroendocrine tumors should be differentiated from GISTs because of their hyperenhancing nature in radiologic images. We report a case of GIST arising in the pancreas that was confirmed by surgical resection.

\section{Introduction}

Gastrointestinal stromal tumor (GIST) is the most common neoplasm of the gastrointestinal tract (GIT) and starts in the interstitial cells of Cajal, which are special cells in the wall of 


\section{Case Reports in Gastroenterology}

Case Rep Gastroenterol 2018;12:671-678

DOI: $10.1159 / 000494553$

(c) 2018 The Author(s). Published by S. Karger AG, Basel www.karger.com/crg

Yeo et al.: An Extragastrointestinal Stromal Tumor Originating from the Pancreas

the GIT [1]. Although a few GISTs arise outside the GIT, in nearby areas such as the omentum, mesentery, or retroperitoneum, GIST arising from the pancreas is rare, with less than 25 cases published to date [2]. As its enhancing nature in radiological images mimics neuroendocrine tumors (NETs), histological confirmation is required for further therapeutic plan. Recently, endoscopic ultrasound (EUS)-guided tissue sampling has been frequently performed for the cytopathologic evaluation of pancreatic solid lesions. We report a case of a pancreatic mass mimicking a NET initially and later diagnosed as GIST using EUS-guided fine-needle aspiration (EUS-FNA) and surgical resection.

\section{Case Presentation}

A 45-year-old woman without any significant medical history presented with a 1-week duration of ambiguous abdominal pain. She visited a local clinic and underwent abdominal computed tomography (CT). A mass at the head of the pancreas was suspected on CT finding. Contrast-enhanced abdominal CT demonstrated an approximately $6.5-\mathrm{cm}$ hypervascular mass with a smooth border, in the head and uncinate process of the pancreas. No signs of dilation of the biliary or pancreatic ducts were found (Fig. 1a, b). She was referred to our hospital after the diagnosis that the mass was suspected of NET or GIST, for further evaluation and management of the pancreatic mass. Physical examination revealed good nutritional status. Laboratory tests at admission demonstrated a normal serum amylase/lipase level of 71/77 U/L. The carbohydrate antigen $19-9$ level was $8.09 \mathrm{U} / \mathrm{mL}$.

Magnetic resonance imaging (MRI) of the pancreas revealed a 6.2-cm mass with a lobulated contour at the pancreatic head, which showed a homogeneous high-signal intensity, restricted diffusion, and low-signal intensity on the T2-weighted image, and high b value (800 $\mathrm{s} / \mathrm{mm}^{2}$ ) on both the diffusion- and pre-contrast T1-weighted images (Fig. 2a-c). In the late arterial phase, early hyperenhancement was observed in the periphery of the mass and enlarged tumor vessel in the center (Fig. 2d). The diagnosis of MRI was islet cell tumor of the pancreas head. The mass of the pancreas was about $6.5 \mathrm{~cm}$ in size, but on CT and MRI, there was no reason to suspect pancreatic cancer. For further evaluation of the pancreatic mass, EUS was performed. During scanning of the pancreas from the duodenum using a linear-array echoendoscope (GF-UCT260; Olympus Optical Co., Tokyo, Japan), a well-defined hypoechoic and heterogeneous mass was identified in the pancreatic uncinate (Fig. 3a). For the differentiation of the enhancing nature of the pancreatic mass, CE-EUS was performed after intravenous injection of 2.4-mL SonoVue (Bracco SpA, Milan, Italy). On real-time imaging, the lesion showed hyperenhancement with a heterogeneous avascular spot as compared with the enhancement of the surrounding pancreatic parenchyma during the arterial and venous phases (Fig. 3c, d). With this pattern, CE-EUS alone could not distinguish between GIST and NET. For an accurate diagnosis, EUS-FNA was performed using a 22-gauge needle (EchoTip; Cook Medical Inc., Winston-Salem, NC, USA). Microscopic examination of a cell block of an acquired FNA specimen showed spindle cell proliferation without significant nuclear enlargement and mitotic activity, which was suggestive of spindle cell tumor (Fig. 4a). The patient underwent pylorus-preserving pancreaticoduodenectomy. Grossly, the surgically resected specimen measured $6.8 \times 4.7$ $\mathrm{cm}$ and was a well-circumscribed solid mass containing focal hemorrhage and a necrosis component (Fig. 4b). Microscopic examination revealed a neoplastic proliferation composed of a spindle cell morphology (Fig. 4c). The mitotic count was $<1$ per 50 high-power fields (HPFs). Immunohistochemistry revealed strong positivity for c-KIT (CD117), DOG-1 (Fig. 4d, e), and 
CD 34 but negativity for S100 (Fig. 4f) and SMA. The Ki67 (Fig. 4g) labelling index was 3\%. She was followed up 6 and 12 months later and was well without any sign of recurrence.

\section{Discussion}

GIST is the most common mesenchymal tumor. It is defined as a c-KIT (CD117)-positive mesenchymal spindle cell or epithelioid tumor in the GIT, omentum, and mesentery [3]. These tumors most commonly occur in the stomach, with a frequency of $60-70 \%$ of all GISTs, followed by $20-25 \%$ in the small intestine, $5 \%$ in the colon and rectum, and $<5 \%$ in the esophagus [4]. Rarely, GIST occurs in the liver, gallbladder, pancreas, and bladder, and is called extragastrointestinal stromal tumor (EGIST), which accounts for $<5 \%$ of all GISTs [5, 6]. A previous report indicated that $<5 \%$ of EGIST cases occur in the pancreas [7]. Less than $1 \%$ of all pancreatic masses are reported as GISTs [4, 8]. GISTs have been thought to originate from the interstitial cells of Cajal in the GIT. If they can occur in other organs, the presence of a subset of GISTs in other cells or the presence of Cajal cells within that organ can be considered [9]. Studies on the differences between GIST and EGIST are not well known because EGIST is rare. According to one study, EGIST has more similar tendencies with GIST in the small intestine than with GIST in the stomach [10].

As GIST is a tumor with spindle cell proliferation, it needs to be differentiated from leiomyoma, schwannoma, inflammatory fibroid polyps, and fibromatosis. GIST can be diagnosed using histopathological examination and immunochemical staining. The c-KIT (CD117) immunohistochemistry test result was positive in $95 \%$ of cases but negative in $5 \%$. The mutation analysis of KIT and platelet-derived growth factor receptor alpha (PDGFRA) is useful for confirming GIST, particularly in cases where CD117 is negative. In addition, the mitotic index is used as a prognostic factor. Mutation analysis is also used as a predictive tool for prognosis and is useful for predicting therapeutic response to imatinib. EUS-FNA is also useful for histopathological diagnosis. The diagnostic rate of luminal GIST through EUS-FNA is known to be $38-89 \%$ [11]. The diagnostic accuracy of EUS-FNA for solid pancreatic lesions is as high as $75-96 \%$ and was reported to be higher for adenocarcinomas than for other solid lesions [12]. Even for benign lesions like in our case, after obtaining sufficient cells by repeated fine-needle aspiration, immunochemical staining can improve the diagnostic accuracy and differential diagnosis [13]. In addition, CE-EUS proved to be a useful technique for differential diagnosis of solid lesions of the pancreas [14, 15]. It is known that CE-EUS pattern can distinguish pancreatic disease [15-20]. In the case of typical pancreatic ductal adenocarcinoma (PDAC), a hypovascular heterogeneous mass with vessel protruding into the mass is seen in CE-EUS [16, 1921]. However, there is a limit to the differentiation of hyperenhanced lesions by CE-EUS. The lesions with hyperenhancement include NET, pancreas metastasis of renal cell carcinoma, and lymphoma. These are the representative diseases that should be distinguished from GIST [16, $19,20]$. The enhancement pattern is difficult to differentiate, and histological examination is necessary.

The patient reported here was referred to our hospital for additional treatment due to suspicion of NET on CT scan performed at a local clinic. NET may occur multiple in the pancreas, CE-EUS was performed to confirm this. Histological examination was performed to exclude malignant tumors and to confirm histologic findings before surgical treatment.

The prognosis of patients with GIST depends on the biological behavior of the disease. Fletcher et al. [22] used tumor size ( $\mathrm{cm}$ ) and mitotic numbers (/50 HPF) to define the criteria for estimating the aggressive behavior and metastatic risk of GIST. According to their criteria, 
GISTs are divided into very low- $(<2 \mathrm{~cm},<5 / 50 \mathrm{HPF})$, low- $(2-5 \mathrm{~cm},<5 / 50 \mathrm{HPF})$, intermediate$(<10 \mathrm{~cm},<5 / 50 \mathrm{HPF})$, and high-risk metastases $(>5 \mathrm{~cm},>5 / 50 \mathrm{HPF}$ or $>10 \mathrm{~cm}$, all mitotic numbers) [22]. Our patient was in the intermediate-risk state at the time of surgery.

The characteristics of GISTs are known to differ depending on the site where they occur. GISTs tend to be more aggressive if they occur from distant parts of the GIT [10]. Most GISTs in the stomach have good prognosis, but those in the small intestine have poor prognosis. Reith et al. [10] reported that nearly half of 48 patients with EGIST died of metastatic or recurrent tumors within an average of 2 years. On the basis of these facts, EGIST is more similar to GIST in the distal GIT. In the present case, the EGIST in the pancreas was surgically removed, and the patient is currently being followed up. However, considering the aggressive tendency of EGIST, even if the patient in our case was in the intermediate-risk state at the time of surgery, a longer, more intensive follow-up would be needed.

As mentioned earlier, EGIST in the pancreas is rare. In the literature review, only 23 cases, including the present case, have been reported. The 23 patients with pancreatic EGIST (11 men and 12 women), with a median age of 55.0 years (range: 30-74), had the following features: the most common clinical features included vague abdominal pain or discomfort, weight loss, and fatigue just like the other pancreatic masses, cancer, NET, and so on. However, 9 cases $(39.0 \%)$ were found incidentally without clinical manifestations. The maximum diameter of these tumors ranged from 2.4 to $35 \mathrm{~cm}$ (median, 9.0). This tumor can occur anywhere in the pancreas. The most common site was the pancreatic head $(8 / 23,33.3 \%)$, followed by the pancreatic tail $(6 / 23,26.1 \%)$, body and tail $(4 / 23,17.4 \%)$, body $(2 / 23,8.7 \%)$, uncinate $(2 / 23,8.7 \%)$, and head and body $(1 / 23,4.3 \%)$. In our review, $69.6 \%$ of pancreatic EGISTs were classified under high-risk categories at the time of diagnosis. Within 30 months of treatment, recurrence occurred in 8 patients (34.8\%), of whom $6(75.0 \%)$ had more frequent metastasis in the liver than in the other sites.

As EGISTs rarely occur in the pancreas, drawing an accurate conclusion about the propensity of pancreatic EGIST is difficult. Overall, these results indicate that pancreatic EGIST tends to be larger and more aggressive than GIST. Therefore, intensive surveillance after surgery is required.

We reported a case of pancreatic stromal tumor diagnosed using EUS-FNA and treated successfully with surgical removal, along with the related literature. Although pancreatic EGIST is rare, prognosis is better than that of PDAC, and contrast enhancement is useful for differentiating it from NET. Therefore, when the mass occurs in the pancreas, the incidence is low, but pancreatic GIST should be considered and EUS-FNA may be used as a diagnostic tool.

\section{Statement of Ethics}

The authors have no ethical conflicts to disclose.

\section{Disclosure Statement}

The authors of this case report declare that they have no competing interests. The authors declare that they have no affiliations with or involvement in any organization or entity with any financial interest or nonfinancial interest (such as personal or professional relationships, affiliations, knowledge, or beliefs) in the subject matter discussed in the manuscript. The 


\section{Case Reports in Gastroenterology}

authors of this case report declare that no financial support nor grant support has been received for the preparation of the manuscript.

\section{References}

1 Blay JY, Bonvalot S, Casali P, Choi H, Debiec-Richter M, Dei Tos AP, et al.; GIST consensus meeting panelists. Consensus meeting for the management of gastrointestinal stromal tumors. Report of the GIST Consensus Conference of 20-21 March 2004, under the auspices of ESMO. Ann Oncol. 2005 Apr;16(4):566-78.

2 Elgeidie A, El-Magd EA, El-Maaty SR, El-Hawary AK. Pancreatic gastrointestinal stromal tumor: a case report. Int J Surg Case Rep. 2016;29:67-70.

3 Miettinen M, Lasota J. Gastrointestinal stromal tumors-definition, clinical, histological, immunohistochemical, and molecular genetic features and differential diagnosis. Virchows Arch. 2001 Jan;438(1):1-12.

4 Daum O, Klecka J, Ferda J, Treska V, Vanecek T, Sima R, et al. Gastrointestinal stromal tumor of the pancreas: case report with documentation of KIT gene mutation. Virchows Arch. 2005 Apr;446(4):470-2.

5 Tian YT, Liu H, Shi SS, Xie YB, Xu Q, Zhang JW, et al. Malignant extra-gastrointestinal stromal tumor of the pancreas: report of two cases and review of the literature. World J Gastroenterol. 2014 Jan;20(3):863-8.

6 Serin KR, Keskin M, Güllüoğlu M, Emre A. An atypically localized gastrointestinal stromal tumor: a case report of pancreas gastrointestinal stromal tumor. Ulus Cerrahi Derg. 2013 Mar;29(1):42-4.

7 Soufi M, Bouziane M, Massrouri R, Chad B. Pancreatic GIST with pancreas divisum: A new entity. Int J Surg Case Rep. 2013;4(1):68-71.

8 Hashimoto M, Tanimoto A, Yoshiya T, Matoba S, Sawada T, Watanabe G, et al. Mesenchymal tumor of the pancreas: report of a case. Surg Today. 2007;37(12):1117-9.

9 Miettinen M. Are desmoid tumors kit positive? Am J Surg Pathol. 2001 Apr;25(4):549-50.

10 Reith JD, Goldblum JR, Lyles RH, Weiss SW. Extragastrointestinal (soft tissue) stromal tumors: an analysis of 48 cases with emphasis on histologic predictors of outcome. Mod Pathol. 2000 May;13(5):577-85.

11 Williams DB, Sahai AV, Aabakken L, Penman ID, van Velse A, Webb J, et al. Endoscopic ultrasound guided fine needle aspiration biopsy: a large single centre experience. Gut. 1999 May;44(5):720-6.

12 Harewood GC, Wiersema MJ. Endosonography-guided fine needle aspiration biopsy in the evaluation of pancreatic masses. Am J Gastroenterol. 2002 Jun;97(6):1386-91.

13 Ando N, Goto H, Niwa Y, Hirooka Y, Ohmiya N, Nagasaka T, et al. The diagnos is of GI stromal tumors with EUS-guided fine needle aspiration with immunohistochemical analysis. Gastrointest Endosc. 2002 Jan;55(1):37-43.

14 Fujita M, Itoi T, Ikeuchi N, Sofuni A, Tsuchiya T, Ishii K, et al. Effectiveness of contrast-enhanced endoscopic ultrasound for detecting mural nodules in intraductal papillary mucinous neoplasm of the pancreas and for making therapeutic decisions. Endosc Ultrasound. 2016 Nov-Dec;5(6):377-83.

15 Götzberger M. [Contrast enhanced endosonography (CE-EUS) for the characterization of small solid pancreatic tumors]. Z Gastroenterol. 2013 Nov;51(11):1327-8.

16 Jang SI, Lee DK. Contrast-enhanced endoscopic ultrasonography: advance and current status. Ultrasonography. 2014 Jul;33(3):161-9.

17 Kanamori A, Hirooka Y, Itoh A, Hashimoto S, Kawashima H, Hara K, et al. Usefulness of contrast-enhanced endoscopic ultrasonography in the differentiation between malignant and benign lymphadenopathy. Am J Gastroenterol. 2006 Jan;101(1):45-51.

18 Sakamoto H, Kitano M, Matsui S, Kamata K, Komaki T, Imai H, et al. Estimation of malignant potential of GI stromal tumors by contrast-enhanced harmonic EUS (with videos). Gastrointest Endosc. 2011 Feb;73(2):227-37.

19 Yamashita Y, Kato J, Ueda K, Nakamura Y, Kawaji Y, Abe H, et al. Contrast-Enhanced Endoscopic Ultrasonography for Pancreatic Tumors. BioMed Res Int. 2015;2015:491782.

20 Park JS, Kim HK, Bang BW, Kim SG, Jeong S, Lee DH. Effectiveness of contrast-enhanced harmonic endoscopic ultrasound for the evaluation of solid pancreatic masses. World J Gastroenterol. 2014 Jan;20(2):518-24.

21 Pei Q, Zou X, Zhang X, Chen M, Guo Y, Luo H: Diagnostic value of EUS elastography in differentiation of benign and malignant solid pancreatic masses: a meta-analysis. Pancreatology: Official Journal of the International Association of Pancreatology (IAP)[et al] 2012;12:402-408.

22 Fletcher CD, Berman JJ, Corless C, Gorstein F, Lasota J, Longley BJ, et al. Diagnosis of gastrointestinal stroma tumors: A consensus approach. Hum Pathol. 2002 May;33(5):459-65. 
Yeo et al.: An Extragastrointestinal Stromal Tumor Originating from the Pancreas
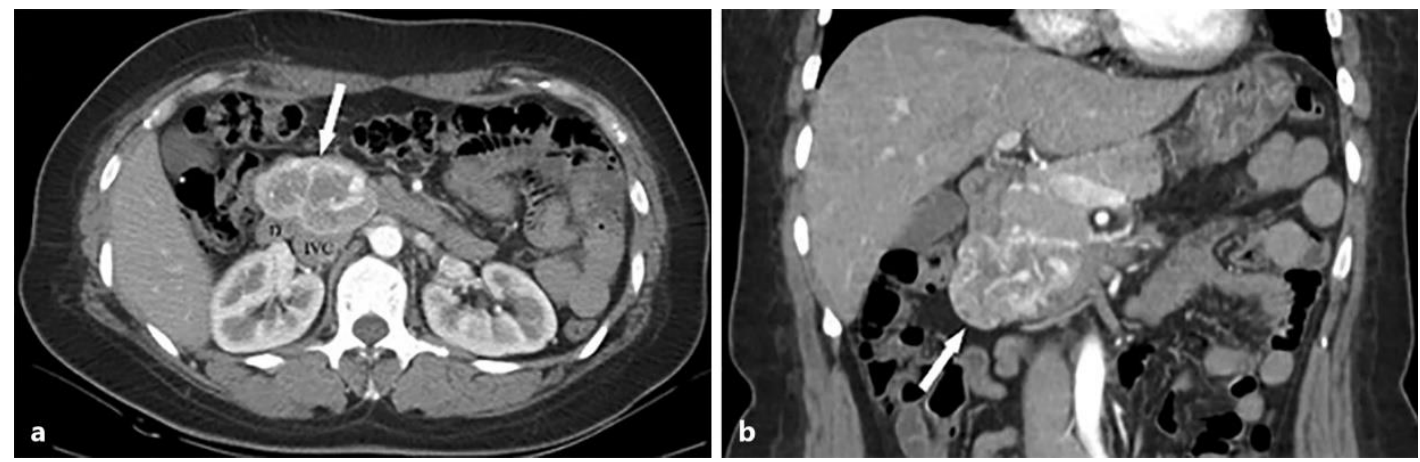

Fig. 1. Abdominal CT findings. a, b An approximately 6.5-cm hypervascular mass with a smooth border (white arrows) can be observed in the head and uncinate process of the pancreas. The mass is closely abutting the duodenum and the IVC. D, duodenum; IVC, inferior vena cava.
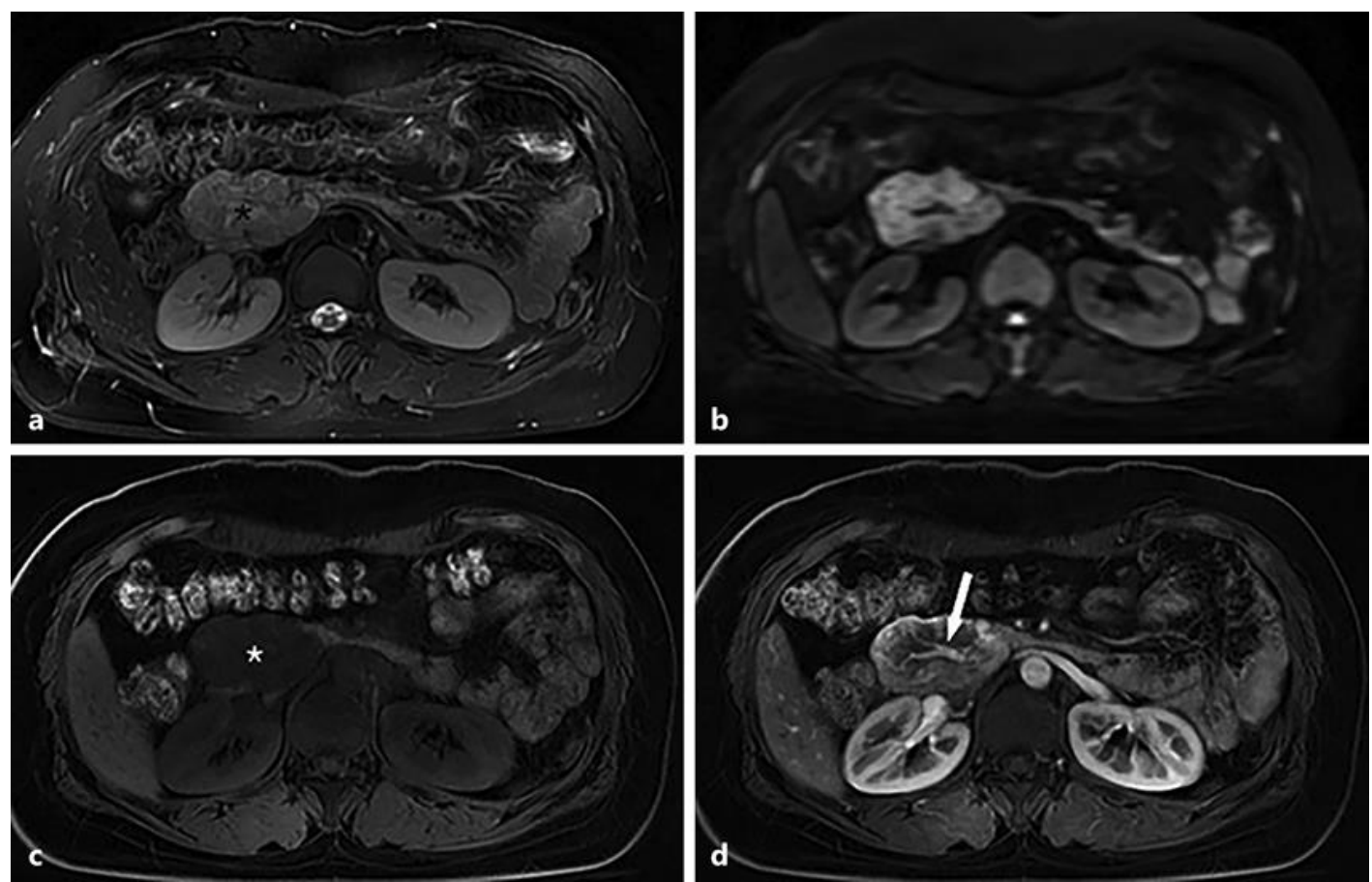

Fig. 2. MRI findings. a The soft tissue mass (black asterisk) shows high signal intensity on the T2-weighted image, without any hemorrhage or necrosis. $\mathbf{b}$ The diffusion-weighted image with a high $\mathbf{b}$ value $(800$ $\mathrm{s} / \mathrm{mm}^{2}$ ) shows restricted diffusion in the mass. $\mathrm{c}$ The mass on pre-contrast T1-weighted image shows low signal intensity (white asterisk). $\mathbf{d}$ In the late arterial phase, early hyperenhancement can be observed in the periphery of the mass and enlarged tumor vessel in the center (white arrow). 

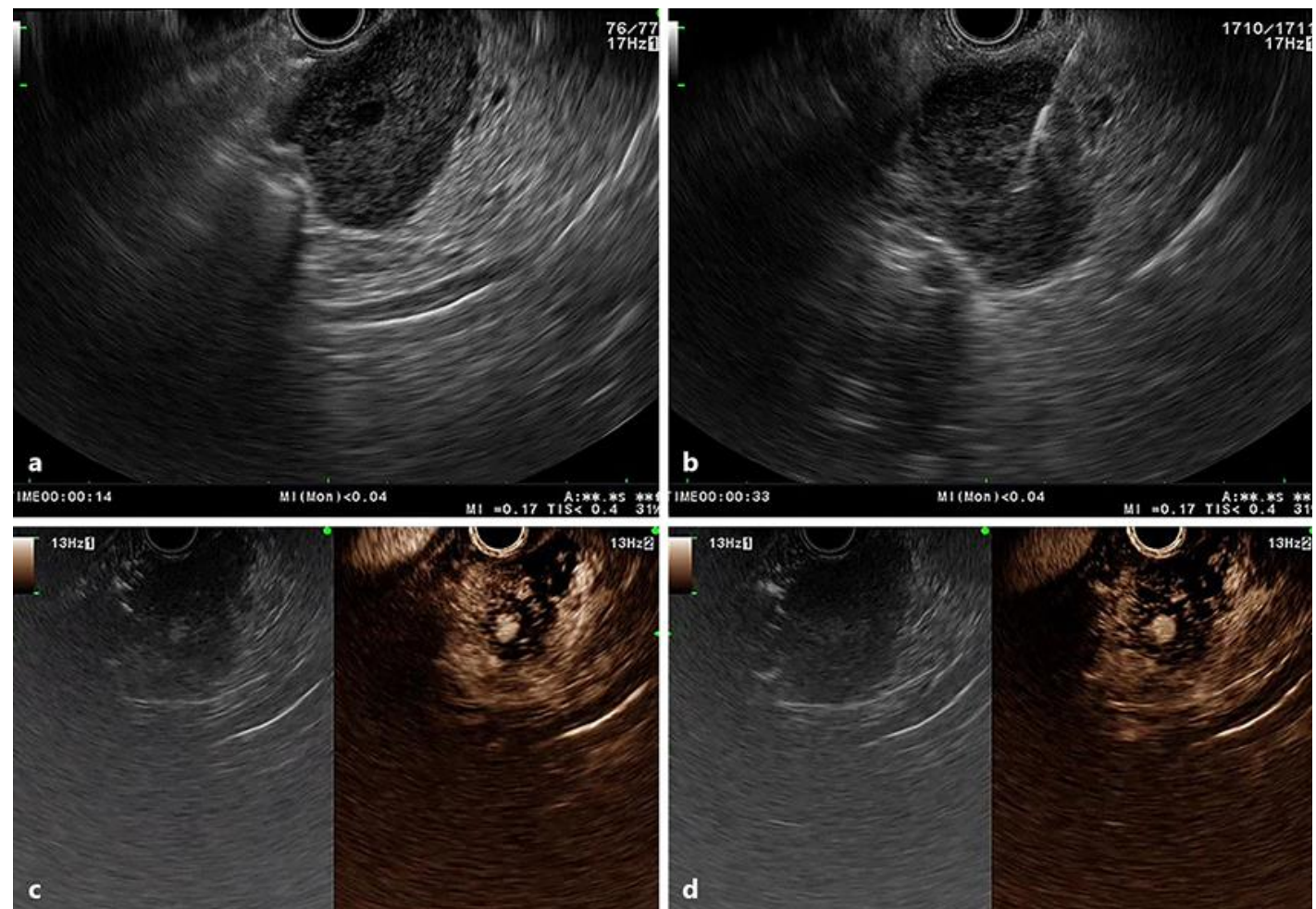

Fig. 3. a A round mass at the uncinate of the pancreas can be seen. The mass is hypoechoic and heterogeneous. The mass measures $36 \times 26 \mathrm{~mm}$ in maximal cross-sectional diameter. Its endoscopic borders are well-defined. $\mathbf{b}$ Diagnostic needle aspiration of the mass was performed. Color Doppler imaging was used prior to needle puncture to confirm a lack of significant vascular structures within the needle path. Three passes were made with a 22-gauge needle using a transduodenal approach. $\mathbf{c}$, $\mathbf{d}$ On the contrast-enhanced harmonic images, the lesion shows heterogeneous hyperenhancement during the arterial and venous phases. 


\section{Case Reports in Gastroenterology}
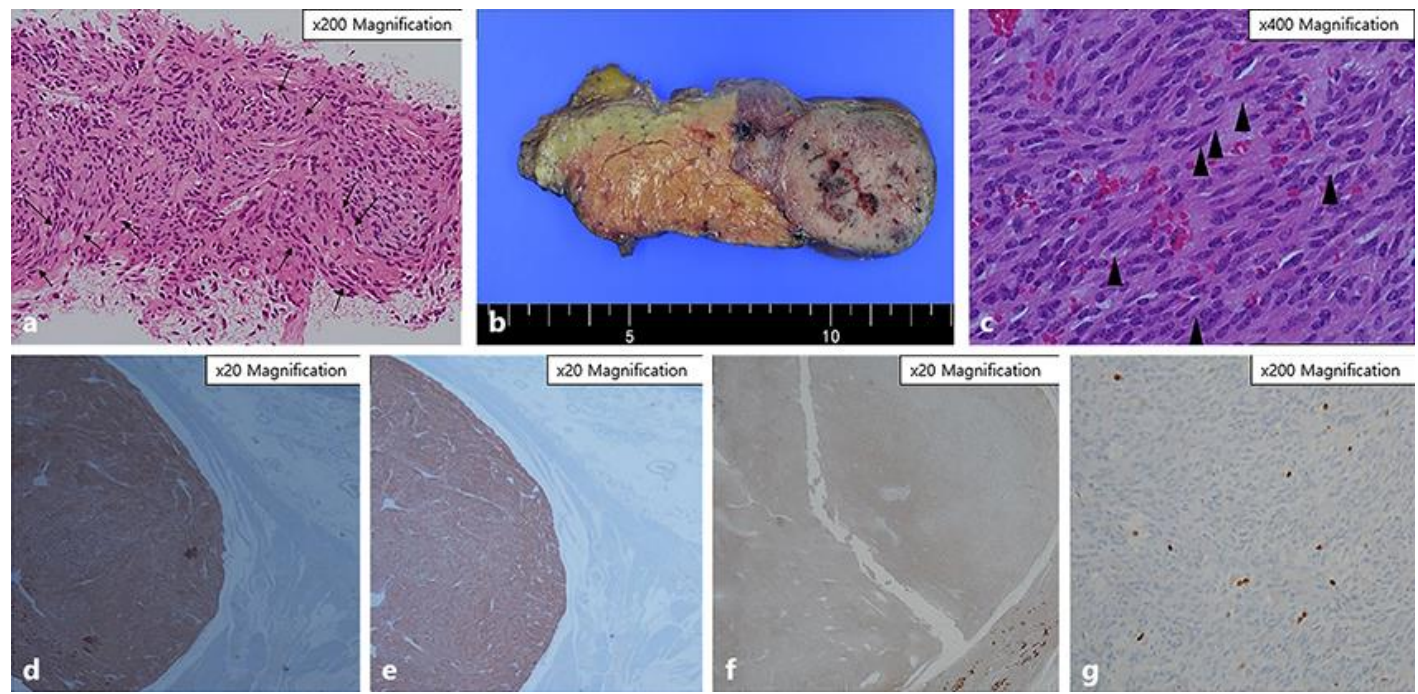

Fig. 4. Pathological findings of the EGIST in the pancreas. a Hematoxylin and eosin (H\&E) staining $(\times 200)$. Cytologic examination of the EUS-FNA specimen showed spindle cells with elongated nuclei (arrows). b The surgically resected specimen shows a brownish to light yellowish solid mass with a well-defined margin. $c$ The lesion shows neoplastic proliferation composed of spindle cells (H\&E staining $\times 400$, arrowheads). $\mathbf{d}$ The spindle cells strongly express c-KIT (CD117) $(\times 20)$ and DOG-1 $(\times 20 ; \mathbf{e}) . \mathbf{f}$ In the S100 staining $(\times 20)$, the spindle cells show negativity. $\mathrm{g}$ The Ki67 $(\times 200)$ labelling index shows $3 \%$. 